[1] J. L. Bentley, Communicated by David Johnson.

[2] D. Bienstock, M. X. Goemans, D. Simchi-Levi and D. P. Williamson. A note on the prize collecting traveling salesman problem. Math. programming, 59 (3): 413-420, 1993.

[3] N. Christofides. Worst-case analysis of a new heuristic for the traveling salesman problem. Report 388, Graduate School of Industrial Administration, Carnegie Mellon University, 1975.

[4] G. Das and P. J. Heffernan. Constructing degree-3 spanners with other sparseness properties. Proc. 4th Annual Intl. Symp. on Algorithms and Computation, LNCS 762, pp. 11-20, December 1993.

[5] D-Z. Du and F. K. Hwang. A proof of the Gilbert-Pollak conjecture on the Steiner ratio. Algorithmica, 7(2): 121-136, 1992.

[6] D-Z. Du, Y. Zhang and Q. Feng. On better heuristic for Euclidean Steiner minimum trees. Proc. 32nd Annual Symp. on Foundations of Comp. Sci, pp. 431-439, October 1991.

[7] T. Fischer. Optimizing the degree of minimum weight spanning trees. TR 93-1338, Dept. of Computer Science, Cornell University, April 1993.

[8] G. N. Frederickson and J. JáJá. On the relationship between the biconnectivity augmentation and traveling salesman problems. Theoret. Comp. Sci., 19 (2): 189-201, 1982.

[9] M. Fürer and B. Raghavachari. Approximating the minimum-degree Steiner tree to within one of optimal. To appear in J. Algorithms. A preliminary version of this paper appeared as "Approximating the minimum degree spanning tree to within one from the optimal degree," in Proc. 3rd Annual ACM-SIAM Symp. on Discrete Algorithms, pp. 317-324, January 1992.

[10] M. R. Garey and D. S. Johnson. Computers and intractability: a guide to the theory of NPcompleteness. Freeman, San Francisco, 1979.

[11] M. Goemans and D. Williamson. A general approximation technique for constrained forest problems. Proc. 3rd Annual ACM-SIAM Symp. on Discrete Algorithms, pp. 307-316, January 1992.

[12] A. Itai, C. H. Papadimitriou and J. L. Szwarcfiter. Hamilton paths in grid graphs. SIAM J. Comput., 11(4): 676-686, 1982.

[13] J. N. Lillington. Some extremal properties of convex sets. Math. Proc. Cambridge Philosophical Society, 77: 515-524, 1975.

[14] C. Monma and S. Suri, Transitions in geometric minimum spanning trees. Discrete \& Computational Geometry, 8(3): 265-293, 1992.

[15] C. H. Papadimitriou and U. V. Vazirani. On two geometric problems related to the traveling salesman problem. J. Algorithms, 5: 231-246, 1984.

[16] R. Ravi, M. V. Marathe, S. S. Ravi, D. J. Rosenkrantz and H. B. Hunt III. Many birds with one stone: multi-objective approximation algorithms. Proc. 25th Annual ACM Symp. on the Theory of Computing, pp. 438-447, May 1993.

[17] G. Robins and J. S. Salowe. On the maximum degree of minimum spanning trees. To appear in 10th Annual ACM Symp. on Computational Geometry, June 1994.

[18] J. S. Salowe. Euclidean spanner graphs with degree four. Proc. of the 8th Annual ACM Symp. on Computational Geometry, pp. 186-191, June 1992. 
$v_{k}$ (again, not necessarily in that order). This path together with $P^{\prime}$ will form a path that starts at $v$ and visits all vertices adjacent to $v$. We now show that

$$
w\left(P^{\prime \prime}\right) \leq \frac{5}{3}\left(\overline{v v_{k-2}}+\overline{v v_{k-1}}+\overline{v v_{k}}\right)
$$

This suffices to prove the lemma. Let $P_{1}, \ldots, P_{6}$ be the six possibilities for $P^{\prime \prime}$. Clearly,

$$
w\left(P^{\prime \prime}\right) \leq \frac{1}{6} \sum_{i=1}^{6} w\left(P_{i}\right)
$$

We will prove that

$$
\frac{1}{6} \sum_{i=1}^{6} w\left(P_{i}\right) \leq \frac{5}{3}\left(\overline{v v_{k-2}}+\overline{v v_{k-1}}+\overline{v v_{k}}\right)
$$

This simplifies to

$$
2 \overline{v_{k-2} v_{k-1} v_{k}}+\sum_{i=k-2}^{k} \overline{v_{j} v_{i}} \leq 5\left(\overline{v v_{k-2}}+\overline{v v_{k-1}}+\overline{v v_{k}}\right) .
$$

Notice that if the above equation is not true, we can "shrink" all the $v_{i}(i=$ $k-2, k-1, k)$ until $\overline{v v_{j}}=\overline{v v_{k-2}}=\overline{v v_{k-1}}=\overline{v v_{k}}$. Assume that $\delta=\left(\overline{v v_{k-2}}-\overline{v v_{j}}\right)+$ $\left(\overline{v v_{k-1}}-\overline{v v_{j}}\right)+\left(\overline{v v_{k}}-\overline{v v_{j}}\right)$. This can be done because the r.h.s decreases by $5 \delta$, and the 1.h.s decreases by at most $5 \delta$. If the above equation is not true then it is also not true when the distance from $v$ to all the points is the same. By scaling, we can assume that the distance of the points from $v$ is 1 . We call this a canonical configuration. The following proposition is implied by Lillington's work [13] and helps in completing the proof.

Proposition 4.2. Let $A, B, C$ and $D$ be points on a unit sphere. The function $F=\overline{A B}+\overline{A C}+\overline{A D}+\overline{B C}+\overline{C D}+\overline{B D}$ reaches a maximum value of $4 \sqrt{6}$ when the points $A, B, C$ and $D$ form a regular tetrahedron.

We will now show that (9) is satisfied by the canonical configuration. The left side of (9) can be written as the sum of sides of the tetrahedron formed by points $\left\{v_{k}, v_{k-1}, v_{k-2}, v_{j}\right\}$ and the sum of sides of the triangle formed by points $\left\{v_{k}, v_{k-1}, v_{k-2}\right\}$. These points lie on a sphere whose center is $v$. By Lemma 4.2, the first sum is bounded by $4 \sqrt{6}$. The second sum is bounded by $3 \sqrt{3}$. Hence the left side of $(9)$ is bounded by $4 \sqrt{6}+3 \sqrt{3}$, which is about 14.994 . The right side of $(9)$ is 15 . Hence $(9)$ is satisfied by the canonical configuration and therefore all configurations. This concludes the proof of Lemma 4.1.

Acknowledgments. We thank Andras Bezdek for telling us about [13]. We thank Karoly Bezdek and Bob Connelly for useful discussions and the committee members of STOC '94 for simplifying the proof of Lemma 3.3 and for pointing out [14].

\section{REFERENCES}


As a function of $\epsilon$, function $F$ is a sum of convex functions minus a linear function, and thus is convex. Therefore, $F$ is maximized either when $\overline{v v_{1}}=1$ or when $v_{1}$ is the midpoint of edge $\overline{v_{2} v_{4}}$ ( since $v_{1}$ is on the convex hull, $v_{1}$ can not cross the edge, hence this interval contains all possible values for $\epsilon$ ).

In the first case, all four points lie on a unit circle with center at $v$. For any four such points, it is easily proven using calculus that $\overline{v_{1} v_{2} v_{3} v_{4}}$ is maximized when the four points are the vertices of a square at $4 \sqrt{2} \approx 5.66$. Thus, $F(1)<0$.

In the second case, $\overline{v_{1} v_{2} v_{3} v_{4}}=\overline{v_{2} v_{3} v_{4}}$. As noted previously, this is at most $3 \sqrt{3} \approx$ 5.2. Thus, $F(\epsilon)<0$.

We now deal with the case when $v_{3}$ is the furthest point. In this case we take the paths $P_{1}=\left[v_{4}, v_{1}, v, v_{2}, v_{3}\right]$ and $P_{2}=\left[v_{3}, v_{4}, v, v_{1}, v_{2}\right]$. The path $P$ added by the algorithm is at most as heavy as the lighter of the paths $P_{1}$ and $P_{2}$. Hence,

$$
w(P) \leq \min \left(P_{1}, P_{2}\right) \leq \frac{w\left(P_{1}\right)+w\left(P_{2}\right)}{2} .
$$

Simplifying, we get

$$
\overline{v_{1} v_{2} v_{3} v_{4}} \leq \frac{1}{2} \overline{v v_{1}}+\frac{5}{2} \overline{v v_{3}}+\frac{3}{2}\left(\overline{v v_{2}}+\overline{v v_{4}}\right) .
$$

The proof of this is identical to the proof of the previous case.

4. Points in higher dimensions. We show how to compute a degree- 3 tree $\left(T_{3}\right)$ when the points are in arbitrary dimension $d \geq 3$. The algorithm for computing the tree is similar to the algorithm for computing degree three trees in the plane - the tree $T_{3}$ is formed by rooting the MST and taking the union of the paths $\left\{P_{v}\right\}$, where each $P_{v}$ is the shortest path starting at $v$ and visiting all of the children of $v$ in the rooted MST. It is known that any Euclidean MST has constant degree [17], so that the algorithm still requires only linear time. The bound on the weight of $T_{3}$ is similar, except that $v$ may have more children. We prove that regardless of the number of children that $v$ has, the weight of $P_{v}$ is at most $5 / 3$ the weight of the edges that it replaces:

LEMma 4.1. Let $\left\{v, v_{1}, v_{2}, \ldots, v_{k}\right\}$ be a set of arbitrary points in $\Re^{d}$. There is a path $P$, starting at $v$, that visits all the points $v_{1}, v_{2}, \ldots, v_{k}$ such that

$$
w(P) \leq \frac{5}{3} \sum_{i=1}^{k} \overline{v v_{i}} .
$$

Proof. We prove this by induction on the degree of $v$. Sort the points in increasing distance from $v$ as $v_{1}, \ldots, v_{k}$. Let $v=v_{0}$. The lemma is trivially true when $k=0,1,2$. Let us assume that the lemma is true for all values of $k$ up to some $\ell \geq 2$. Consider $k=\ell+1$. By the induction hypothesis, the claim is true when $v$ has $k-3$ children; hence we can find a path $P^{\prime}$ that starts at $v$ and visits all vertices $v_{i}(i=1, \ldots, k-3)$ (not necessarily in that order) such that $w\left(P^{\prime}\right) \leq \frac{5}{3} \sum_{i=1}^{k-3} \overline{v v_{i}}$. Let $v_{j}$ be the last vertex on the path $P^{\prime}$. We add the cheapest path $P^{\prime \prime}$ that starts at $v_{j}$ and visits $v_{k-2}, v_{k-1}$ and 
Further simplifying, we get:

$$
\overline{v_{1} v_{2} v_{3} v_{4}} \leq \frac{1}{2} \overline{v v_{1}}+\frac{5}{2} \overline{v v_{4}}+\frac{3}{2}\left(\overline{v v_{2}}+\overline{v v_{3}}\right) .
$$

Note that if it happens that $v_{3}$ was the farthest point from $v$, among its children, we get a similar equation with $v_{3}$ and $v_{4}$ being exchanged in r.h.s of the equation. By symmetry, the case when $v_{2}$ is furthest is similar to $v_{4}$ being farthest.

Without loss of generality, $\overline{v v_{3}} \geq \overline{v v_{2}}$. The proof now proceeds in a manner similar to the proof of Lemma 3.3. If there is a configuration of points for which this equation is not true (the 1.h.s exceeds the r.h.s) then we can move $v_{4}, v_{3}$ closer to $v$ until $\overline{v v_{2}}=$ $\overline{v v_{3}}=\overline{v v_{4}}$. In doing this, we decrease the 1.h.s by at most $2\left(\overline{v v_{4}}-\overline{v v_{2}}\right)+2\left(\overline{v v_{3}}-\overline{v v_{2}}\right)$. Clearly, the r.h.s decreases by exactly $4\left(\overline{v v_{4}}-\overline{v v_{2}}\right)+4\left(\overline{v v_{3}}-\overline{v v_{2}}\right)$. This ensures that the 1.h.s is still greater than the r.h.s. Hence without loss of generality, if there is a configuration for which our equation is not true then there is a configuration with the property that $\overline{v v_{4}}=\overline{v v_{3}}=\overline{v v_{2}}$. We now show that when this property is true there is no counter-example.

By scaling, we may assume that $\overline{v v_{4}}=\overline{v v_{3}}=\overline{v v_{2}}=1$, and $\overline{v v_{1}}=\epsilon$, where $\epsilon \leq 1$.

Note that (by Corollary 3.2) $v$ was originally within the convex hull of its four children. Also (by Corollary 3.2), every child is on the convex hull. These properties are both maintained by the above shrinking steps.

We now wish to prove that

$$
\overline{v_{1} v_{2} v_{3} v_{4}} \leq \frac{11}{2}+\frac{1}{2} \epsilon .
$$

It is easily shown using elementary calculus that for any $\epsilon$ such that $v_{1}$ is on the convex hull of the points $\left\{v_{1}, \ldots, v_{4}\right\}$, rotating $v_{1}$ and $v_{3}$ around $v$ until $\angle v_{1} v v_{2}=\angle v_{1} v v_{4}$ (see Fig. 9) and $\angle v_{2} v v_{3}=\angle v_{4} v v_{3}$ does not decrease the perimeter. Also, it maintains that $v_{1}$ is on the convex hull. Assume the two pairs of angles are equal, and define $F(\epsilon)=\overline{v_{1} v_{2} v_{3} v_{4}}-\epsilon / 2-11 / 2$. We will show $F$ is non-positive over the range of possible $\epsilon$.

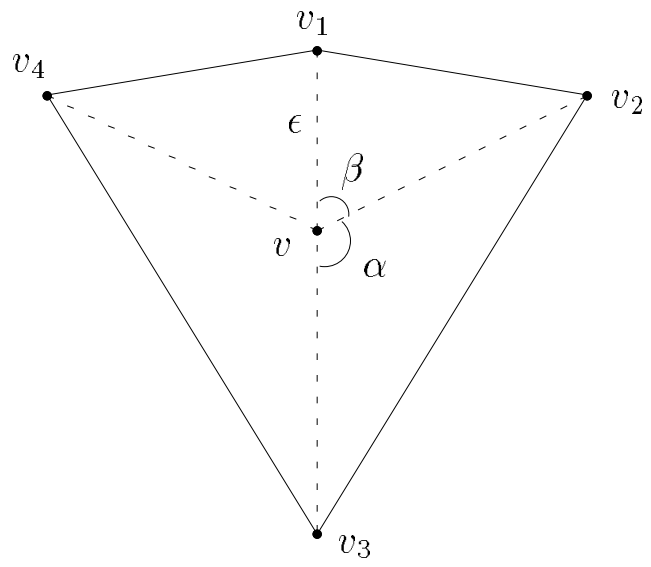

Fig. 9. Figure to illustrate degree four case. 
and hence

$$
\begin{aligned}
\overline{v_{1} v_{2} v_{3}} & \leq(\sqrt{3}-1) \overline{v v_{1}}+(2 \sqrt{3}-3) \overline{v v_{1}}+2\left(\overline{v v_{2}}+\overline{v v_{3}}\right) \\
& \leq(\sqrt{3}-1) \overline{v v_{1}}+\left(\sqrt{3}+\frac{1}{2}\right)\left(\overline{v v_{2}}+\overline{v v_{3}}\right) .
\end{aligned}
$$

This proves $(7)$.

Case 2: $v$ has \& children, $v_{1}, v_{2}, v_{3}, v_{4}$. Assume that $v_{1}$ is the point that is closest to $v$, among its children. Let the order of the points be $v_{1}, v_{2}, v_{3}, v_{4}$, when we scan the plane clockwise from $v$, starting from an arbitrary direction.

There are two cases, depending on whether $v_{4}$ or $v_{3}$ is the point that is furthest from $v$ among its children. We first address the case when $v_{4}$ is the furthest point. (The proof for the case when $v_{2}$ is the point furthest from $v$ is symmetric to the case when $v_{4}$ is the furthest point.)

Consider the following paths (see Fig. 8): $P_{1}=\left[v_{4}, v_{1}, v, v_{2}, v_{3}\right]$ and $P_{2}=\left[v_{4}, v_{3}, v, v_{1}, v_{2}\right]$.

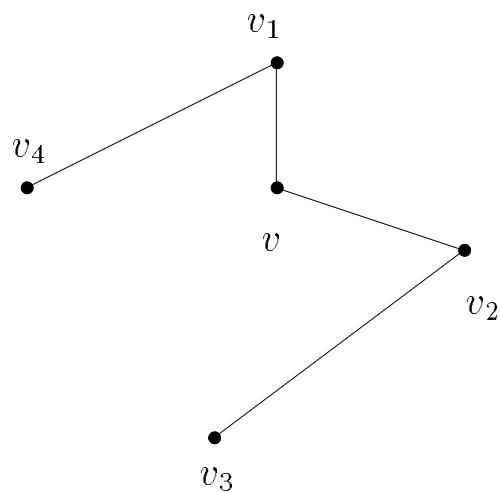

$P_{1}$

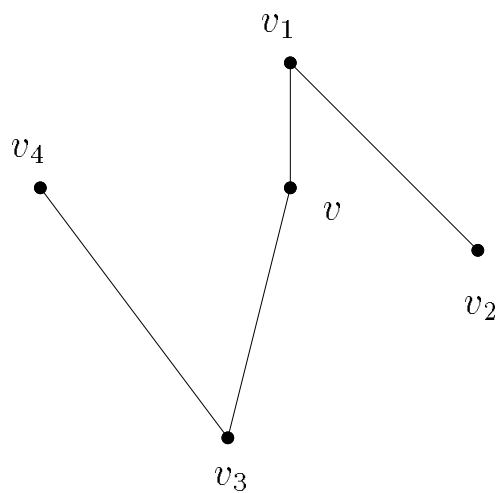

$P_{2}$

FIG. 8. T4, four children

The path $P_{v}$ added by the algorithm is at most as heavy as the lighter of the paths $P_{1}$ and $P_{2}$. Hence

$$
w\left(P_{v}\right) \leq \min \left(P_{1}, P_{2}\right) \leq \frac{w\left(P_{1}\right)+w\left(P_{2}\right)}{2}
$$

We will show that

$$
\frac{w\left(P_{1}\right)+w\left(P_{2}\right)}{2} \leq 1.25\left(\overline{v v_{1}}+\overline{v v_{2}}+\overline{v v_{3}}+\overline{v v_{4}}\right) .
$$

Simplifying, we need to show that

$$
\frac{1}{2}\left(\overline{v_{4} v_{1}}+\overline{v_{1} v}+\overline{v v_{2}}+\overline{v_{2} v_{3}}+\overline{v_{4} v_{3}}+\overline{v_{3} v}+\overline{v v_{1}}+\overline{v_{1} v_{2}}\right) \leq \frac{5}{4}\left(\overline{v v_{1}}+\overline{v v_{2}}+\overline{v v_{3}}+\overline{v v_{4}}\right) .
$$


Theorem 3.9. Let $T$ be a minimum spanning tree of a set of points in $\Re^{2}$. Let $T_{4}$ be the spanning tree output by the algorithm in Fig. 6 .

$$
w\left(T_{4}\right) \leq 1.25 \times w(T) .
$$

Proof of Lemma 3.8. The proof is similar to the proof of Lemma 3.5. As before, we consider cases depending on the number of children of $v$. The cases when $v$ has no children, one child, or two children are trivial.

Case 1: $v$ has 3 children, $v_{1}, v_{2}, v_{3}$. Let $v_{1}$ be the point that is closest to $v$, among its children. Consider the following four paths (see Fig. 7): $P_{1}=\left[v_{2}, v_{1}, v, v_{3}\right], P_{2}=$ $\left[v_{2}, v, v_{1}, v_{3}\right], P_{3}=\left[v_{1}, v, v_{2}, v_{3}\right]$ and $P_{4}=\left[v_{1}, v, v_{3}, v_{2}\right]$.

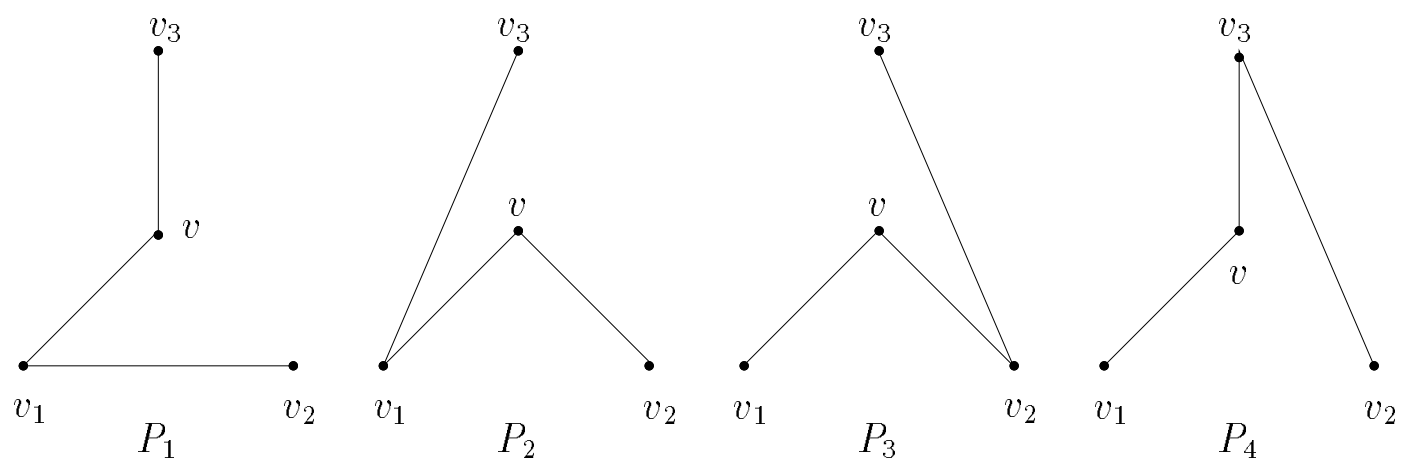

Fig. 7. $T_{4}$, three children

Clearly,

$$
w\left(P_{v}\right) \leq \frac{w\left(P_{1}\right)}{3}+\frac{w\left(P_{2}\right)}{3}+\frac{w\left(P_{3}\right)}{6}+\frac{w\left(P_{4}\right)}{6} .
$$

We will show that

$$
\frac{w\left(P_{1}\right)}{3}+\frac{w\left(P_{2}\right)}{3}+\frac{w\left(P_{3}\right)}{6}+\frac{w\left(P_{4}\right)}{6} \leq \frac{2+\sqrt{3}}{3}\left(\overline{v v_{1}}+\overline{v v_{2}}+\overline{v v_{3}}\right) .
$$

This proves the three-child case because $\frac{2+\sqrt{3}}{3}$ approximately equals 1.244 and is less than 1.25. This simplifies to

$$
\frac{\overline{v_{1} v_{2}}+\overline{v_{1} v_{3}}+\overline{v_{2} v_{3}}}{3}+\overline{v v_{1}}+\frac{\overline{v v_{2}}+\overline{v v_{3}}}{2} \leq \frac{2+\sqrt{3}}{3}\left(\overline{v v_{1}}+\overline{v v_{2}}+\overline{v v_{3}}\right),
$$

which further simplifies to

$$
\overline{v_{1} v_{2} v_{3}} \leq(\sqrt{3}-1) \overline{v v_{1}}+\left(\sqrt{3}+\frac{1}{2}\right)\left(\overline{v v_{2}}+\overline{v v_{3}}\right) .
$$

Since $v_{1}$ is the closest point to $v$, applying Lemma 3.3, we get

$$
\overline{v_{1} v_{2} v_{3}} \leq(3 \sqrt{3}-4) \overline{v v_{1}}+2\left(\overline{v v_{2}}+\overline{v v_{3}}\right) .
$$




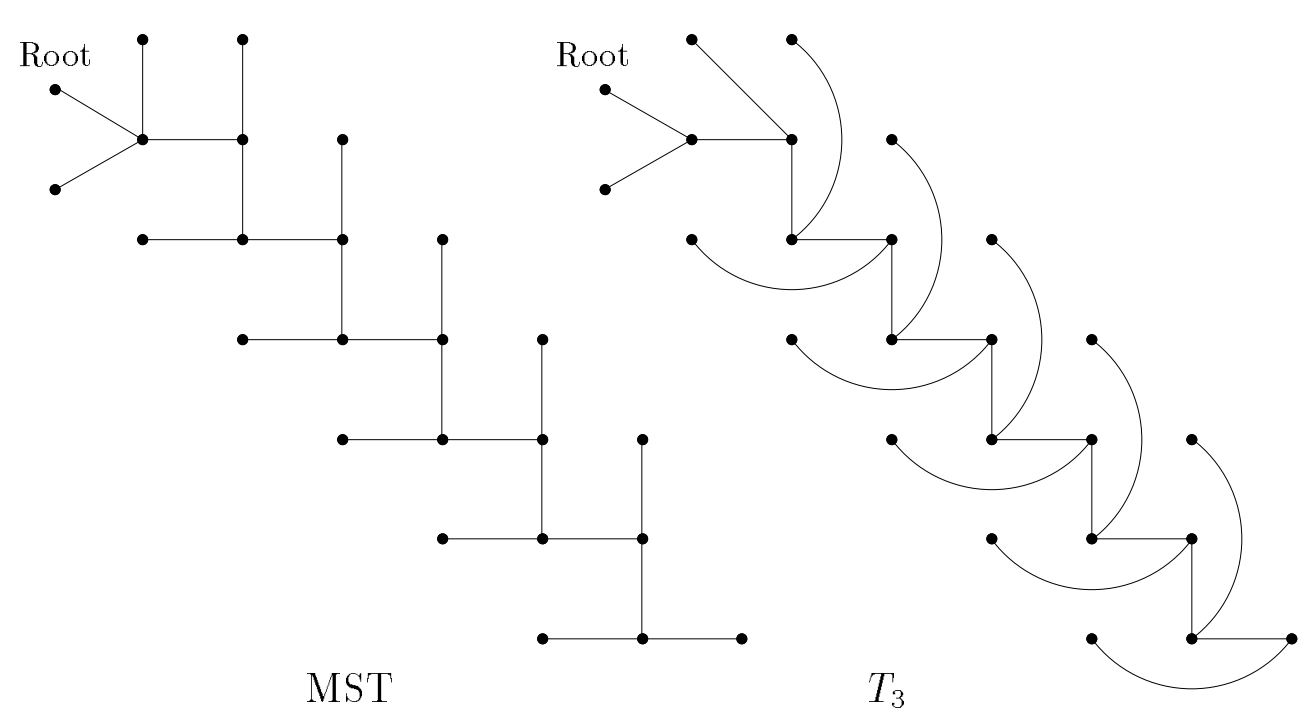

FIG. 5. Bad example for algorithm in Fig. 2.

High Level Description: The basic idea is the same as in the previous algorithm.

The difference is that we don't insist that each path $P_{v}$ start at $v$. The tree is rooted at an arbitrary leaf. For each vertex $v$, the minimum weight path $P_{v}$ visiting $v$ and all of $v$ 's children (not necessarily starting at $v$ ) is computed. The final tree $T_{4}$ consists of the union of the paths $\left\{P_{v}\right\}$. Again, for the analysis we think of each path $P_{v}$ replacing the edges between $v$ and its children in $T$.

Tree-4 $(V, T)$ - Find a degree 4 tree of $V$.

1 Root the MST $T$ at a leaf vertex $r$.

2 For each vertex $v \in V$ do

3 Compute the shortest path $P_{v}$ visiting $v$ and all its children.

4 Return $T_{4}$, the tree formed by the union

of the paths $\left\{P_{v}\right\}$.

Fig. 6. Algorithm to find a degree 4 tree.

LEMm A 3.7. The algorithm in Fig. 6 returns a degree-4 spanning tree of the given set of points $V$.

Proof. A proof by induction shows that $T_{4}$ is a tree. Each vertex $v$ occurs in at most two paths and thus has degree at most four.

Lemma 3.8. Let $v$ be a vertex in an MST T for a set of points in $\Re^{2}$. Let $P_{v}$ be the shortest path visiting $\{v\} \cup \operatorname{child}_{T}(v)$.

$$
w\left(P_{v}\right) \leq 1.25 \times \sum_{v_{i} \in \operatorname{child}_{T}(v)} \overline{v v_{i}} .
$$

From the above lemma, each path $P_{v}$ weighs at most 1.25 times the net weight of the edges it replaces. Thus, 
We want to show that the function $F(\epsilon)=\overline{v_{1} v_{2} v_{3} v_{4}}+\left(\overline{v_{1} v_{2}}+\overline{v_{1} v_{4}}\right)-7-3 \epsilon$ is non-positive in the range $0 \leq \epsilon \leq 1$. Simplifying, we get

$$
F(\epsilon)=2 \overline{v_{1} v_{2}}+\overline{v_{2} v_{3}}+\overline{v_{3} v_{4}}+2 \overline{v_{1} v_{4}}-7-3 \epsilon .
$$

Each of $\overline{v_{i} v_{j}}$ in the definition of $F$ is a convex function of $\epsilon$ due to the following reason. Let $p$ be the point closest to $v_{j}$ on the line connecting $v_{i}$ and $v^{\prime}$. Observe that as $v_{i}$ moves towards $v^{\prime}, \overline{v_{i} v_{j}}$ decreases if $v_{i}$ is moving towards $p$ and increases otherwise. Since $F$ is a sum of convex functions minus a linear function, it is a convex function of $\epsilon$. Therefore it is maximized at either $\epsilon=0$ or $\epsilon=1$.

When $\epsilon=1$, all four points are at the same distance from $v^{\prime}$. If angle $\angle v_{4} v^{\prime} v_{1}=$ $\alpha$ then $F$ can be written as a function of a single variable $\alpha$ and it can be verified that $F$ reaches a maximum value of $10 \sqrt{0.8}-10$, which is non-positive.

When $\epsilon=0, \overline{v_{1} v_{2}}=\overline{v_{1} v_{4}}=1$. Simplifying we get $F=\overline{v_{2} v_{3}}+\overline{v_{3} v_{4}}-3$, and it reaches a maximum value of $2 \sqrt{2}-3$, which is non-positive (when $\epsilon=0$, note that $v_{1}$ is the midpoint of the line segment $v_{2} v_{4}$ ).

Case $3 b . v^{\prime} v_{2}=\epsilon$. We wish to prove that

$$
\overline{v_{1} v_{2} v_{3} v_{4}}+\left(\overline{v_{1} v_{2}}+\overline{v_{1} v_{4}}\right) \leq 8+2 \epsilon .
$$

We want to show that the function $F^{\prime}(\epsilon)=\overline{v_{1} v_{2} v_{3} v_{4}}+\left(\overline{v_{1} v_{2}}+\overline{v_{1} v_{4}}\right)-8-2 \epsilon$ is non-positive in the range $0 \leq \epsilon \leq 1$.

As a function of $\epsilon$, function $F^{\prime}$ is a sum of convex functions minus a linear function, and thus is convex. Therefore it is maximized at either $\epsilon=0$ or $\epsilon=1$.

The case $\epsilon=1$ leads to the same configuration as in Case 3a.

When $\epsilon=0, \overline{v_{1} v_{2}}=\overline{v_{2} v_{3}}=1$. Here $F^{\prime}=2 \overline{v_{1} v_{4}}+\overline{v_{3} v_{4}}-5$. If angle $\angle v_{4} v^{\prime} v_{1}=\alpha$, then $F^{\prime}$ can be written as a function of a single variable $\alpha$ and it can be verified that $F^{\prime}$ reaches a maximum value of $5 \sqrt{0.8}-5$, which is non-positive.

This concludes the proof of Lemma 3.5.

The example in Fig. 5 shows that the 1.5 factor is tight for the algorithm in Fig. 2, modified according to the note following its description. The same example also shows that the 1.5 factor is tight for the unmodified algorithm since the unmodified algorithm never outputs a lighter tree than the modified algorithm. Each curved arc shown in Fig. 5 is actually a straight line, and has been drawn curved for convenience. The vertex that is the child of the root has three children, and is forced to drop one child. In doing so, the degree of its child goes to four, and it in turn drops one of its children. The algorithm could make choices in such a way that the changes propagate through the tree and the tree $T_{3}$ output by the algorithm may be as shown in the figure. The ratio of the cost of the final solution to the cost of the MST can be made arbitrarily close to 1.5 .

3.3. Spanning trees of degree four. We now assume that we are given a Euclidean minimum spanning tree in which every vertex has degree at most 5 . We show how to convert this tree to a tree in which every vertex has degree at most 4. 


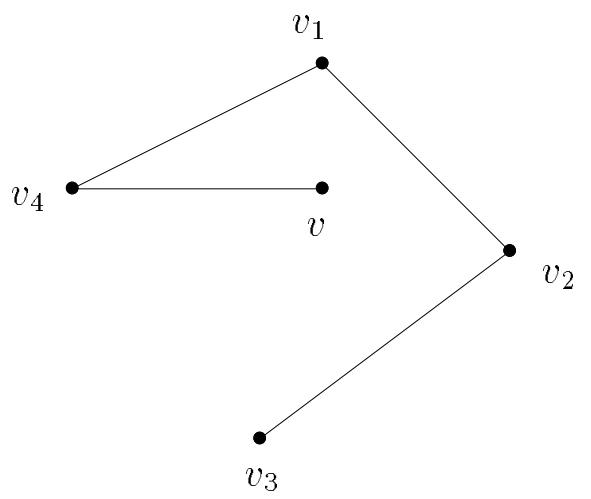

$P_{1}$

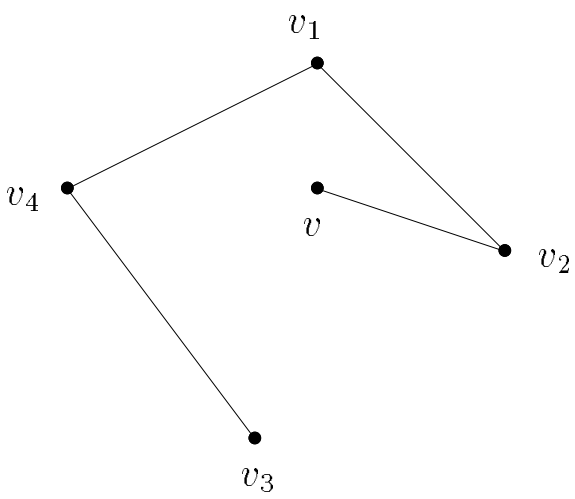

$P_{2}$

FIG. 4. $T_{3}$, four children

We will show that

$$
\frac{1}{2}\left(w\left(P_{1}\right)+w\left(P_{2}\right)\right) \leq 1.5\left(\overline{v v_{1}}+\overline{v v_{2}}+\overline{v v_{3}}+\overline{v v_{4}}\right)
$$

This simplifies to

$$
\overline{v_{1} v_{2} v_{3} v_{4}}+\left(\overline{v_{1} v_{2}}+\overline{v_{1} v_{4}}\right) \leq 3\left(\overline{v v_{1}}+\overline{v v_{3}}\right)+2\left(\overline{v v_{2}}+\overline{v v_{4}}\right)
$$

We will first prove that

$$
\overline{v_{1} v_{2} v_{3} v_{4}}+\left(\overline{v_{1} v_{2}}+\overline{v_{1} v_{4}}\right) \leq 3\left(\overline{v^{\prime} v_{1}}+\overline{v^{\prime} v_{3}}\right)+2\left(\overline{v^{\prime} v_{2}}+\overline{v^{\prime} v_{4}}\right)
$$

Once we prove $(6)$, by triangle inequality we can conclude that (5) is true. (Since $\overline{v v_{1}}+\overline{v v_{3}} \geq \overline{v_{1} v_{3}}=\overline{v^{\prime} v_{1}}+\overline{v^{\prime} v_{3}}$ and $\overline{v v_{2}}+\overline{v v_{4}} \geq \overline{v_{2} v_{4}}=\overline{v^{\prime} v_{2}}+\overline{v^{\prime} v_{4}}$.)

We prove $(6)$ by contradiction. Suppose there exists a set of points which does not satisfy (6). Suppose we shrink $v^{\prime} v_{3}$ by $\delta$. The left side of the above inequality decreases by at most $2 \delta$, whereas the right side of the inequality decreases by exactly $3 \delta$. Therefore as we shrink $v^{\prime} v_{3}$, the inequality stays violated. Suppose $v^{\prime} v_{3}$ shrinks and becomes equal to another edge $v^{\prime} v_{i}$ for some $i \in\{1,2,4\}$. We now shrink both $v^{\prime} v_{3}$ and $v^{\prime} v_{i}$ simultaneously at the same rate. Again it is easy to show that the inequality continues to be violated as $v^{\prime} v_{3}$ and $v^{\prime} v_{i}$ shrink. Hence we reach a configuration where three of the edges are equal.

Without loss of generality, the length of the three edges is 1 and the length of the fourth edge is some $\epsilon \leq 1$.

There are two cases to consider. The first is when $v^{\prime} v_{1}=\epsilon$ and the second is when $v^{\prime} v_{2}=\epsilon$. (The case when $v^{\prime} v_{4}=\epsilon$ is the same as the second case.)

Case $3 a \cdot v^{\prime} v_{1}=\epsilon$. We wish to prove that

$$
\overline{v_{1} v_{2} v_{3} v_{4}}+\left(\overline{v_{1} v_{2}}+\overline{v_{1} v_{4}}\right) \leq 7+3 \epsilon .
$$


Case 1: $v$ has 2 children, $v_{1}, v_{2}$. There are two possible paths for $P_{v}$, namely $P_{1}=$ $\left[v, v_{1}, v_{2}\right]$ and $P_{2}=\left[v, v_{2}, v_{1}\right]$. Clearly,

$$
w\left(P_{v}\right)=\min \left(w\left(P_{1}\right), w\left(P_{2}\right)\right) \leq \frac{w\left(P_{1}\right)+w\left(P_{2}\right)}{2}=\frac{\overline{v v_{1}}}{2}+\frac{\overline{v v_{2}}}{2}+\overline{v_{1} v_{2}} \leq 1.5\left(\overline{v v_{1}}+\overline{v v_{2}}\right) .
$$

Case 2: $v$ has 3 children, $v_{1}, v_{2}, v_{3}$. Let $v_{1}$ be the child that is nearest to $v$. Consider the following four paths (see Fig. 3): $P_{1}=\left[v, v_{1}, v_{2}, v_{3}\right], P_{2}=\left[v, v_{1}, v_{3}, v_{2}\right], P_{3}=\left[v, v_{2}, v_{1}, v_{3}\right]$ and $P_{4}=\left[v, v_{3}, v_{1}, v_{2}\right]$.
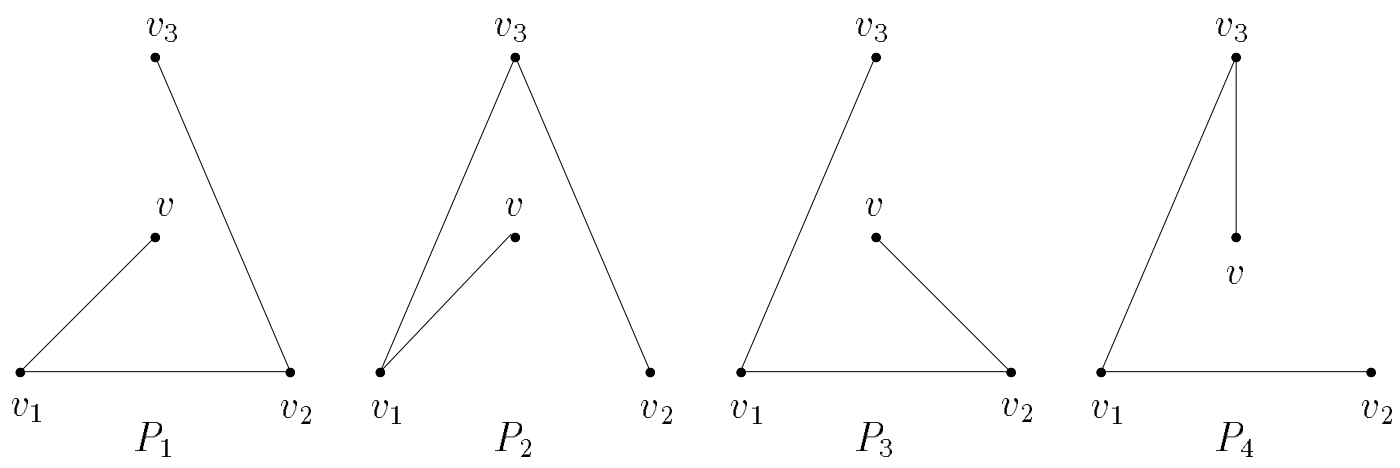

FIG. 3. $T_{3}$, three children

The path $P_{v}$ is at most as heavy as the lightest of $\left\{P_{1}, P_{2}, P_{3}, P_{4}\right\}$. The weight of the lightest of these paths is at most any convex combination of the weights of the paths. Specifically,

$$
w\left(P_{v}\right) \leq \min \left(w\left(P_{1}\right), w\left(P_{2}\right), w\left(P_{3}\right), w\left(P_{4}\right)\right) \leq \frac{w\left(P_{1}\right)}{3}+\frac{w\left(P_{2}\right)}{3}+\frac{w\left(P_{3}\right)}{6}+\frac{w\left(P_{4}\right)}{6} .
$$

We will now prove that

$$
\frac{w\left(P_{1}\right)}{3}+\frac{w\left(P_{2}\right)}{3}+\frac{w\left(P_{3}\right)}{6}+\frac{w\left(P_{4}\right)}{6} \leq 1.5\left(\overline{v v_{1}}+\overline{v v_{2}}+\overline{v v_{3}}\right) .
$$

This simplifies to

$$
\overline{v_{1} v_{2}}+\overline{v_{2} v_{3}}+\overline{v_{3} v_{1}} \leq 1.25 \overline{v v_{1}}+2\left(\overline{v v_{2}}+\overline{v v_{3}}\right)
$$

which follows from Lemma 3.3.

Case 3: $v$ has \& children, $v_{1}, v_{2}, v_{3}, v_{4}$. Let $v^{\prime}$ be the point of intersection of the diagonals $\overline{v_{1} v_{3}}$ and $\overline{v_{2} v_{4}}$. Note that the diagonals do intersect because the polygon $v_{1} v_{2} v_{3} v_{4}$ is convex (follows from Corollary 3.2).

Let $v_{3}$ be the point that is furthest from $v^{\prime}$, among $\left\{v_{1}, v_{2}, v_{3}, v_{4}\right\}$. Consider the following two paths (see Fig. 4): $P_{1}=\left[v, v_{4}, v_{1}, v_{2}, v_{3}\right], P_{2}=\left[v, v_{2}, v_{1}, v_{4}, v_{3}\right]$.

Clearly,

$$
w\left(P_{v}\right) \leq \min \left(w\left(P_{1}\right), w\left(P_{2}\right)\right) \leq \frac{w\left(P_{1}\right)}{2}+\frac{w\left(P_{2}\right)}{2}
$$


3.2. Spanning trees of degree three. We now assume that we are given a Euclidean minimum spanning tree $T$ of degree at most five. We show how to convert $T$ into a tree of degree at most three. The weight of the resulting tree is at most 1.5 times the weight of $T$.

High Level Description: The tree $T$ is rooted at an arbitrary leaf vertex. Since $T$ is a degree- 5 tree, once it is rooted at a leaf, each vertex has at most four children. For each vertex $v$, the shortest path $P_{v}$ starting at $v$ and visiting every child of $v$ is computed. The final tree $T_{3}$ consists of the union of the paths $\left\{P_{v}\right\}$. Fig. 2 gives the above algorithm. In analyzing the algorithm, we think of each vertex $v$ as replacing its edges from its children with the path $P_{v}$.

TrEe-3(V,T) - Find a degree 3 tree of $V$.

1 Root the MST $T$ at a leaf vertex $r$.

2 For each vertex $v \in V$ do

3 Compute $P_{v}$, the shortest path starting at $v$ and visiting all the children of $v$.

4 Return $T_{3}$, the tree formed by the union

of the paths $\left\{P_{v}\right\}$.

FIG. 2. Algorithm to find a degree 3 tree.

Note: Typically, the initial MST has very few nodes with degree greater than three [1]. In practice, it is worth modifying the algorithm to scan the vertices in preorder, maintaining the partial tree $T_{3}$ of edges added so far, and to add paths to $T_{3}$ as follows. When considering a vertex $v$, if the degree of $v$ in the partial $T_{3}$ is two, add the path $P_{v}$ as described in the algorithm. Otherwise its degree is one and, in this case, relax the requirement that the added path must start at $v$. That is, add the shortest path that visits $v$ and all of $v$ 's children to $T_{3}$ (see $\S 3.3$ ). This modification will never increase the cost of the resulting tree, but may offer substantially lighter trees in practice.

LEMм A 3.4. The algorithm in Fig. 2 outputs a spanning tree of degree three.

Proof. An easy proof by induction shows that the union of the paths forms a tree. Each vertex $v$ is on at most two paths and is an interior vertex of at most one path.

LEMma 3.5. Let $v$ be a vertex in an MST T of a set of points in $\Re^{2}$. Let $P_{v}$ be a shortest path visiting $\{v\} \cup$ child $_{T}(v)$ with $v$ as one of its endpoints.

$$
w\left(P_{v}\right) \leq 1.5 \times \sum_{v_{i} \in \operatorname{child}_{T}(v)} \overline{v v_{i}}
$$

By the above lemma, each path $P_{v}$ has weight at most 1.5 times the weight of the edges it replaces. Thus,

THEOREM 3.6. Let $T$ be a minimum spanning tree of a set of points in $\Re^{2}$. Let $T_{3}$ be the spanning tree output by the algorithm in Fig. 2.

$$
w\left(T_{3}\right) \leq 1.5 \times w(T) .
$$

Proof of Lemma 3.5. We consider the various cases that arise depending on the number of children of $v$. The cases when $v$ has no children or exactly one child are trivial. 
The lemma is also interesting in its own right and we believe that it and the associated techniques will be useful in other geometrical problems.

Lemma 3.3. Let $X, A, B$, and $C$ be points in $\Re^{d}$ with $\overline{X A} \leq \overline{X B}, \overline{X C}$. Then

$$
\overline{A B C} \leq(3 \sqrt{3}-4) \overline{X A}+2(\overline{X B}+\overline{X C}) .
$$

Note that $3 \sqrt{3}-4 \approx 1.2$. Recall that $\overline{A B C}$ is the perimeter of the triangle and $\overline{X Y}$ is the distance from $X$ to $Y$.

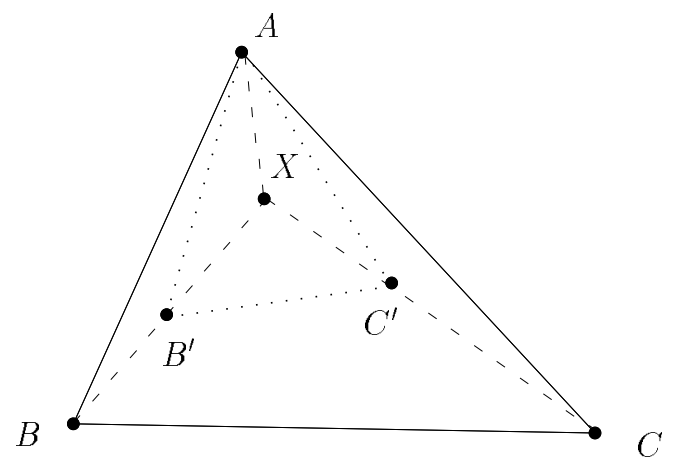

FIG. 1. Shrinking to obtain canonical form

Proof. Let $B^{\prime}$ and $C^{\prime}$ be points on $X B$ and $X C$ respectively such that $\overline{X A}=$ $\overline{X B^{\prime}}=\overline{X C^{\prime}}$ (see Fig. 1). First we observe that the lemma is true if it is true for the points $X, A, B^{\prime}$ and $C^{\prime}$. This follows because by triangle inequality,

$$
\overline{A B C} \leq \overline{A B^{\prime} C^{\prime}}+2 \overline{B B^{\prime}}+2 \overline{C C^{\prime}} .
$$

By our assumption,

$$
\overline{A B^{\prime} C^{\prime}} \leq(3 \sqrt{3}-4) \overline{X A}+2\left(\overline{X B^{\prime}}+\overline{X C^{\prime}}\right) .
$$

Combining the two inequalities yields the desired result. Therefore in the rest of the proof, we show that the lemma is true when the "arms" $\overline{X A}, \overline{X B^{\prime}}$ and $\overline{X C^{\prime}}$ are equal.

It is not very difficult to see that to maximize the perimeter of the triangle, $X$ will be in the plane defined by $A, B^{\prime}$ and $C^{\prime}$, and thus $X$ is at the center of a circle passing through $A, B^{\prime}$ and $C^{\prime}$.

By scaling, it suffices to consider the case when the circle has unit radius. In this case, the right-hand side of (4) is exactly $3 \sqrt{3}$. Thus, it suffices to show that the maximum perimeter achieved by any triangle whose vertices lie on a unit circle is $3 \sqrt{3}$. This is easily proved [13].

Note that in an arbitrary metric space it is possible to have an (equilateral) triangle of perimeter six and a point $X$ at distance one from each vertex. 
or a Steiner tree of a given subset of vertices in a graph, with degree at most one more than minimum was given by Fürer and Raghavachari [9]. This was extended to weighted graphs by Fischer [7]. He shows how to find minimum spanning trees whose degree is within a constant multiplicative factor plus an additive $O(\log n)$ of the optimal degree. The degree bound is improved further in the case when the number of different edge weights is bounded by a constant. Ravi, Marathe, Ravi, Rosenkrantz and Hunt [16] consider the problem of computing bounded-degree subgraphs satisfying given connectivity properties in a graph whose edge weights satisfy the triangle inequality. They give efficient algorithms for computing subgraphs which have low weight and small bottleneck cost. Salowe [18], and Das and Heffernan [4] consider the problem of computing bounded-degree graph spanners and provide algorithms for computing them. Robins and Salowe [17] study the maximum degrees of minimum spanning trees under various metrics.

2. Preliminaries. Let $V=\left\{v_{1}, \ldots, v_{n}\right\}$ be a set of $n$ points in the plane. Let $G$ be the complete graph induced by $V$, where the weight of an edge is the Euclidean distance between its endpoints. We use the terms points and vertices interchangeably. Let $\overline{u v}$ be the Euclidean distance between vertices $u$ and $v$. Let $T_{\min }$ be a minimum spanning tree (MST) of the points in $V$. Let $w(T)$ denote the total weight of a spanning tree $T$. Let $T_{k}$ denote a spanning tree in which every vertex has degree at most $k$. Let $\operatorname{deg}_{T}(v)$ be the degree of a vertex $v$ in the tree $T$. Let $\triangle A B C$ denote the triangle formed by points $A, B$ and $C$. Let $\angle A B C$ denote the angle formed at $B$ between line segments $A B$ and $B C$. Let $\overline{A B C}$ denote the perimeter of $\triangle A B C$.

In this paper we prove the following: for an arbitrary set of points in $\Re^{2}$,

$$
\begin{aligned}
& \exists T_{3}: \quad w\left(T_{3}\right) \leq 1.5 \times w\left(T_{\min }\right) \\
& \exists T_{4}: \quad w\left(T_{4}\right) \leq 1.25 \times w\left(T_{\min }\right)
\end{aligned}
$$

For an arbitrary set of points in $\Re^{d}(d>2)$,

$$
\exists T_{3}: \quad w\left(T_{3}\right) \leq \frac{5}{3} \times w\left(T_{\min }\right)
$$

3. Points in the plane. We first consider the case of $\Re^{2}$ - points in the plane. We first note some useful properties of minimum spanning trees in $\Re^{d}$.

Proposition 3.1 ([15]). Let $A B$ and $B C$ be two edges incident to a point $B$ in a minimum spanning tree of a set of points in $\Re^{d}$. Then $\angle A B C$ is a largest angle in $\triangle A B C$.

Corollary 3.2. Let $A B$ and $B C$ be two edges incident to a point $B$ in a minimum spanning tree of a set of points in $\Re^{d}$. Then

- $\angle A B C \geq 60^{\circ}$

- $\angle B A C, \angle B C A \leq 90^{\circ}$.

3.1. An upper bound on the perimeter of a triangle. We now prove an upper bound on the perimeter of an arbitrary triangle in terms of distances to its vertices from an arbitrary point. This lemma is useful in proving the performances of our algorithms. 
tree problem. It should be noted that in the special case of $K=2$, Christofides [3] gave a simple and elegant polynomial time approximation algorithm with an approximation ratio of 1.5 for computing a traveling salesperson tour for points satisfying the triangle inequality (points in a metric space).

1.1. Our Contributions. In this paper, we show that for an arbitrary collection of $n$ points in the plane, there exists a degree- 3 spanning tree whose weight is at most 1.5 times the weight of a minimum spanning tree. We also show that there exists a degree-4 spanning tree whose weight is at most 1.25 times the weight of a minimum spanning tree. This solves a ten year old open problem posed by Papadimitriou and Vazirani [15].

Moreover, if a minimum spanning tree is given as part of the input, the trees can be computed in $O(n)$ time. Note that our bound of 1.5 for the degree- 3 spanning tree problem is an "absolute" guarantee (based on the weight of an MST) as opposed to a "relative" guarantee for the degree-2 spanning tree obtained by Christofides [3] (based on the weight of an optimal solution).

We also generalize our results to points in higher dimensions. We show that for any $d \geq 2$, an arbitrary collection of points in $\Re^{d}$ contains a degree- 3 spanning tree whose weight is at most $5 / 3$ times the weight of a minimum spanning tree. This is the first paper that achieves factors better than two for these problems.

1.2. Significance of Our Results. Many approximation algorithms make use of the triangle inequality to obtain approximate solutions to NP-hard problems. These algorithms typically involve a "short-cutting" step where the triangle inequality is used to bound the cost of the obtained solution. Examples include Christofides' heuristic for the traveling salesperson problem [3], biconnectivity augmentation [8], approximate weighted matching [11], prize-collecting traveling salesperson [2], and bounded-degree subgraphs which have low weight and small bottleneck cost [16].

A question of general interest is how to obtain improved approximation algorithms for such problems when the points come from a Euclidean, as opposed to arbitrary, metric space. This requires making use of more than just the triangle inequality. Surprisingly, for most problems, improved algorithms are not known. (A notable exception is the famous Euclidean Steiner tree problem [5, 6].) We use rudimentary geometric techniques to obtain an improved algorithm for the Euclidean degree- $K$ spanning tree problem.

The key to our method is to give short-cutting steps that are provably better than implied by the triangle inequality alone. Lemma 3.3, which bounds the perimeter of an arbitrary triangle in terms of distances to its vertices from any point, is typical of the techniques that we use to get better bounds.

1.3. Related Work. Papadimitriou and Vazirani showed that any MST whose vertices have integer co-ordinates has maximum degree at most five [15]. Monma and Suri [14] showed that for every set of points in the plane, there exists a degree-5 MST.

Many recent works have given algorithms to find subgraphs of bounded degree that simultaneously satisfy other given constraints. An algorithm to find a spanning tree 


\title{
LOW DEGREE SPANNING TREES OF SMALL WEIGHT
}

\author{
SAMIR KHULLER * BALAJI RAGHAVACHARI ${ }^{\dagger}$ AND NEAL YOUNG ${ }^{\ddagger}$
}

\begin{abstract}
Given $n$ points in the plane, the degree- $K$ spanning tree problem asks for a spanning tree of minimum weight in which the degree of each vertex is at most $K$. This paper addresses the problem of computing low-weight degree- $K$ spanning trees for $K>2$. It is shown that for an arbitrary collection of $n$ points in the plane, there exists a spanning tree of degree three whose weight is at most 1.5 times the weight of a minimum spanning tree. It is shown that there exists a spanning tree of degree four whose weight is at most 1.25 times the weight of a minimum spanning tree. These results solve open problems posed by Papadimitriou and Vazirani. Moreover, if a minimum spanning tree is given as part of the input, the trees can be computed in $O(n)$ time.

The results are generalized to points in higher dimensions. It is shown that for any $d \geq 3$, an arbitrary collection of points in $\Re^{d}$ contains a spanning tree of degree three, whose weight is at most $5 / 3$ times the weight of a minimum spanning tree. This is the first paper that achieves factors better than two for these problems.
\end{abstract}

AMS(MOS) subject classification. 05C05, 05C10, 05C85, 65Y25, 68Q20, 68R10, 68U05, 90C27, 90C35.

Key words. algorithms, graphs, spanning trees, approximation algorithms, geometry.

1. Introduction. Given $n$ points in the plane, how do we find a spanning tree of minimum weight among those in which each vertex has degree at most $K$ ? Here the weight of an edge between two points is defined to be the Euclidean distance between them. This problem is referred to as the Euclidean degree- $K$ spanning tree problem and is a generalization of the Hamilton Path problem which is known to be NP-hard [10, 12]. When $K=3$, it was shown to be NP-hard by Papadimitriou and Vazirani [15], who conjectured that it is NP-hard for $K=4$ as well. When $K=5$, the problem can be solved in polynomial time [14].

This paper addresses the problem of computing low weight degree- $K$ spanning trees for $K>2$. In any metric space, it is known that there always exists a spanning tree of degree 2 whose cost is at most twice the cost of a minimum spanning tree (MST). This is shown by taking an Euler tour of an MST (in which each edge is taken twice) and producing a Hamilton tour by short-cutting the Euler tour. In the case of general metric spaces, it is easy to generate examples in which the ratio of a shortest Hamilton path to the weight of a minimum spanning tree is arbitrarily close to two. But such examples do not translate to points in $\Re^{d}$. In view of this, Papadimitriou and Vazirani [15] posed the problem of obtaining factors better than two for the Euclidean degree- $K$ spanning

* Department of Computer Science and Institute for Advanced Computer Studies, University of Maryland, College Park, MD 20742. Research supported by NSF Research Initiation Award CCR9307462. E-mail : samir@cs .umd.edu.

$\dagger$ Department of Computer Science, The University of Texas at Dallas, Box 830688, Richardson, TX 75083-0688. E-mail : rbk@utdallas .edu.

$\ddagger$ School of Operations Research and Industrial Engineering, Cornell University, Ithaca, NY 148533801. Part of this work was done while at UMIACS and was supported in part by NSF grants CCR-8906949 and CCR-9111348. E-mail : ney@orie.cornell.edu. 